\title{
Proper bootstrapping
}

\author{
Igor Douven • Christoph Kelp
}

Received: 1 June 2011 / Accepted: 18 April 2012 / Published online: 4 May 2012

(C) The Author(s) 2012. This article is published with open access at Springerlink.com

\begin{abstract}
According to a much discussed argument, reliabilism is defective for making knowledge too easy to come by. In a recent paper, Weisberg aims to show that this argument relies on a type of reasoning that is rejectable on independent grounds. We argue that the blanket rejection that Weisberg recommends of this type of reasoning is both unwarranted and unwelcome. Drawing on an older discussion in the philosophy of science, we show that placing some relatively modest restrictions on the said type of reasoning suffices to block the anti-reliabilist argument.
\end{abstract}

Keywords Confirmation theory · Bootstrapping - Easy knowledge

According to reliabilists,

Reliabilist Tenet (RT) our beliefs can be known, or at least be warranted, even in the absence of warrant for believing that the sources from which they stem are reliable.

All that matters for warrant, they claim, is that a belief was in fact reliably formed; for knowledge, one just adds the requirement of truth. Backed by RT, reliabilists have an effective response to one of the most familiar types of skeptic, who challenges the epistemic status of our beliefs by questioning the grounds we have for holding these beliefs, and then goes on to question the grounds of whatever we adduce to our defense. This tactic does not get off the ground against the reliabilist, whose answer simply is that our beliefs can be knowledge, or at least be warranted, whether or not we

\footnotetext{
I. Douven $(\varangle)$

Faculty of Philosophy, University of Groningen, Groningen, The Netherlands

e-mail: i.e.j.douven@rug.nl

C. Kelp

Institute of Philosophy, University of Leuven, Leuven, Belgium

e-mail: christoph.kelp@hiw.kuleuven.be
} 
have warrant for believing that they were reliably formed. By these lights, our beliefs having the epistemic status they have is independent of our ability (or inability, for that matter) to provide a satisfactory response to the skeptic's challenges.

Attractive though the position may be, reliabilism has recently come under attack from philosophers who have accused it of making, in some cases, warrant, and even knowledge, too easy to come by. For instance, it has been claimed that if RT were correct, we could come to know that our gas gauge is reliable simply by looking at it on sufficiently many different occasions, which would be absurd since surely more is needed to obtain such knowledge. This is now commonly referred to as the "problem of easy knowledge."

In a recent paper, Weisberg (2009) attempts to show that this is only an apparent problem. More exactly, he argues that the type of reasoning that licenses the acquisition of the problematic easy knowledge-bootstrapping, as the reasoning has come to be called - is faulty, and so may be (and should be) rejected by the reliabilist. While we think Weisberg is right in finding fault with the reasoning involved in the problem of easy knowledge, we would like to argue that he overshoots in declaring bootstrapping generally to be illicit. If, as he thinks, there is independent reason to reject bootstrapping, then that would be bad news, for it seems that the type of reasoning that he wants us to reject is a quite common and arguably indispensable type of scientific reasoning.

Drawing on ideas developed by philosophers of science in the 1980s and 1990s, we show that a much less drastic solution to the problem is available. Specifically, philosophers of science have identified a number of restrictive conditions under which bootstrapping is not to be applied, and it is precisely one of those conditions that is being violated in the reasoning that gives rise to the problem of easy knowledge. So, in order to avoid the problem of easy knowledge, it suffices for the reliabilist to insist on a proper application of bootstrapping; there is no need for a wholesale rejection of it. This conclusion will be supported by showing that the cases Weisberg adduces as putatively providing independent motivation against bootstrapping only discredit an unrestricted application of bootstrapping: they, too, are blocked by the restrictions that have already been proposed in the philosophy of science literature.

We begin by stating more explicitly than above the problem of easy knowledge (Sect. 1), then discuss Weisberg's diagnosis of it, as well as the solution to it that he proposes (Sect. 2), and finally extract an important insight from the discussion of bootstrapping in the philosophy of science and show how it helps us to solve, without having to give up a valuable tool in the scientist's toolbox, both the original problem of easy knowledge and the additional problem cases Weisberg presents (Sect. 3).

\section{The problem of easy knowledge}

To see in a bit more detail how the problem of easy knowledge is generated, consider a person who forms beliefs about the level of fuel in her car's gas tank by looking at the gas gauge. Say that the gas gauge is reliable, and so, according to the reliabilist, is a knowledge source — or at least a source of warrant — for the person, even if she does not know or even warrantedly believe that the gas gauge is reliable. Then the claim is that the person could obtain warrant (at least) for believing that the gas gauge is 
reliable by means of a simple inductive argument, the premises of which are all of the form: "At time $t$ the gauge reads that the tank is $x$ percent full and at time $t$ the tank is $x$ percent full." According to the argument, the reliabilist is committed to the claim that these premises are warranted for the person: the first conjunct of each premise is warranted by her looking at the gas gauge (supposing perception to be reliable) and the second conjunct by the gauge reading and the presumption that the gauge is reliable, which, considering RT, is enough to turn it into a source of warranted belief. So, once the person has warrant for sufficiently many premises of the designated form, and supposing induction to be reliable, an inductive step will warrant her in believing, and will even yield knowledge, that, for any time $t$, if at $t$ the gauge reads that the tank is $x$ percent full, then at $t$ the tank is indeed $x$ percent full. In other words, the person has inductively obtained warrant for believing, and knowledge of, the proposition that the gauge is reliable. ${ }^{1}$

This conclusion should be hard to swallow for anyone, reliabilists not excluded. After all, it is eminently reasonable to think that in order to obtain knowledge about the reliability of the gauge one would, at a minimum, have to check directly how much fuel is in the car's gas tank and to compare this amount with the gauge reading, and to repeat this procedure several times, on different occasions, and for sufficiently many different gauge readings.

This line of thought is often viewed as presenting a dilemma for reliabilism: reliabilists must either give up RT_ - but then, what remains of their swift answer to the skeptic? - or accept the seemingly absurd conclusion of the above argument.

However, some reliabilists have, in accepting the said conclusion, been willing to bite the bullet here. In their view, it is still better that knowledge is sometimes easier to come by than we had thought than it is that knowledge cannot be attained at all, as the skeptic claims (see, e.g., Van Cleve (2003) and Bergmann (2004)); and they see no hope for diverting the skeptical threat unless we stick to RT. However, it goes without saying that even these philosophers would be happier if the above argument against their position could be blocked so that there was no bullet to be bitten in the first place.

Focussing on the other horn of the putative dilemma, Zalabardo (2005) argues that reliabilists can safely reject $\mathrm{RT}$, given that it is compatible with their position to hold that we often do have warrant for believing that a belief source is reliable. This warrant need not consist in having evidence one might be able to cite in response to the skeptic's challenge, so that, as before, an inability to satisfy the skeptic does not undermine the status of our beliefs. However, taking this route requires that the reliabilist is willing to accept "a slight weakening of the sufficient conditions for warrant postulated by [her] account - namely a provision to the effect that a belief source will not count as a knowledge source if the subject believes it to be reliable and this belief has not been formed with a reliable source" (p. 54). Leaving to the side whether Zalabardo is correct in thinking that the resulting position would still be impervious to a skeptical attack, we note that it is at least a bit much to believe that we could jeopardize a belief source's status as a knowledge source merely by forming the belief that the belief source is reliable.

\footnotetext{
${ }^{1}$ We have loosely followed Vogel's (2000, p. $613 \mathrm{ff}$ ) presentation of the argument here.
} 
It is far from clear, however, that the problem of easy knowledge confronts the reliabilist with a dilemma. For how troublesome it really is, and whether it poses a genuine problem for the reliabilist at all, will also depend on how one assesses the inferential steps involved in the problematic type of case. These inferential steps are clearly not trivial; it is not, for instance, as though the argument required no more than two applications of modus ponens and one application of conjunction elimination. So, rather than contemplating whether to accept the first or the second horn of the alleged dilemma, and whether doing so is consistent with maintaining a reliabilist position, reliabilists may focus their attention on whether there is some inferential step in the argument that they could plausibly object to and thus try to slip between the horns of the dilemma.

A prima facie good candidate for this may be the inductive step. But unless we can identify some special reason for ruling induction inapplicable in the present case, the objection would have to be to induction in general, and that would amount to embracing inductive skepticism. While this might still leave us with a sizeable portion of knowledge, it would evidently be a very high price to pay. In fact, it would be insufficient to give up the claim that induction is or at least can be a knowledge producing inferential mechanism. As facing a "problem of easy warrant" would be no less embarrassing than facing the problem of easy knowledge, we would even have to deny the possibility of obtaining warranted belief on the basis of inductive inference. So, not only could we not have any knowledge about the future, we could not have any warranted beliefs about it either.

There might seem to be a less costly option. Notice that the above argument crucially relies on a multi-premise closure principle for warrant, or at least on the principle that warranted belief is closed under conjunction. For the conjuncts of each of the premises of the inductive argument, warrant is acquired separately: the first conjunct (in our presentation) is warranted by the person's looking at the gauge, the second conjunct by something more involved. Some closure principle is needed, then, to ensure that the conjunctions (and not just their conjuncts) are warrantedly credible as well, for the inductive argument takes as input the conjunctions, and not the conjuncts. ${ }^{2}$ So, a second possible target for the reliabilist who wants to escape the conclusion of the above argument is to reject at least the conjunction principle, and thus a full-blown multi-premise closure principle, for warranted belief. ${ }^{3}$

\footnotetext{
2 In the argument one could also go from "At $t$, the gauge reads that the tank is $x$ percent full" and "At $t$, the tank is $x$ percent full," not to their conjunction, but to "At $t$, the gauge reading is correct." Clearly, however, while that would avoid use of the conjunction principle, it would still require some multi-premise closure principle.

3 This might seem to be the obvious way to go for those who already take single-premise closure to generate a problem of easy knowledge. For instance, some authors have argued that, while it would pre-theoretically seem that one can come to know relatively easily that there are zebras in the pen before one, supposing a single-premise closure principle according to which, roughly, we can know the logical consequences of each proposition we know would just as easily yield the further knowledge that there are no cleverly disguised mules in the pen; but the latter piece of knowledge would seem much more difficult to attain than the former (see, e.g., Dretske (1970)). However, notice first that abandoning even single-premise closure would leave no room for deduction as a tool for extending our knowledge or warranted beliefs, which would be odd. Second, an argument very similar to the one given in Douven (2007) in response to Harman's paradox might help to defuse the threat to single-premise closure as well. The gist of the argument would be that, while
} 
But we think there are good reasons for resisting this move, too. Here, we refer the reader to Douven (2002, Sect. 2) for a survey of what we believe to be valid reasons for keeping to the conjunction principle; see Douven and Williamson (2006) for an additional reason. And, as closure under conjunction is generally regarded to be the problematic part of the multi-premise closure principle, we take these reasons also to support a case for keeping aboard multi-premise closure for warranted belief in general. For those who are unmoved by these reasons, we note that it should still be of some interest to see that a rejection of multi-premise closure is in any case not required for blocking the problem of easy knowledge. Above we left open the possibility that there exists a reason for resisting the inductive step in that argument, even if induction in general is valid. Weisberg's recent analysis of the argument, now to be discussed, points to one such reason. Our own analysis, to be presented in Sect. 3, will point to another.

\section{Weisberg on the problem of easy knowledge}

Weisberg proceeds by presenting three additional problem cases that involve reasoning which is pre-theoretically similar to, and supposedly as objectionable as, the reasoning underlying the problem of easy knowledge, then considers what is common to all these cases, and on the basis of this analysis suggests a solution to the problem.

The first additional case is meant not only to problematize bootstrap reasoning but also to establish that the problem of easy knowledge affects internalists as much as reliabilists. ${ }^{4}$ In this case, a person knows that the gas gauge of his car is reliable. As it happens, the gauge is super-reliable, more reliable, that is, than is required (according to reliabilist standards) to grant knowledge, but the person is initially unaware of this. Nevertheless, by the same reasoning that was employed in the original problem of easy knowledge, the person can come to know that the gauge is not only reliable but even super-reliable. This conclusion is intuitively as unacceptable as the conclusion of the problem of easy knowledge. Yet rejecting RT is of no avail here, because the person knows - and so has warrant for believing — that the relevant beliefs were reliably formed.

Vogel (2008, p. 531) may seem to offer a solution to this problem as well as to the original problem of easy knowledge by way of the following principle:

\footnotetext{
Footnote 3 continued

we do not have knowledge, nor a warranted belief, of there being zebras in the pen, we have something very close to knowledge of, as well as something very close to warrant for, that proposition, much closer, at any rate, than any skeptic would be willing to grant us. Still, given that the closure principle for knowledge requires-obviously - knowledge (and not only something very close to knowledge) of, and the closure principle for warrant - just as obviously_warrant (and not only something very close to warrant) for, the premise from which the conclusion is inferred, one cannot easily come to know, or believe warrantedly, that the animals in the pen are not cleverly disguised mules. But this is a topic for another paper.

4 That the problem of easy knowledge is not exclusively a problem for reliabilism was also noted in Cohen (2002), Bergmann (2004), Vogel (2008). But the versions of internalism considered in those papers are still committed to a qualified version of RT (like the claim that sensory perception gives us immediate justification). To our knowledge, Weisberg is the first to argue that even an outright rejection of RT will not solve the problem of easy knowledge.
} 
No Rule Circularity (NRC) A belief that an epistemic rule $R$ is reliable cannot be justified by the application of $R$. That is, neither the conclusion itself nor any belief which supports the conclusion may be justified in virtue of the application of $R$.

Patently, NRC solves the original problem of easy knowledge: in that case, the rule of going by the gas gauge serves to establish that going by the gas gauge is reliable. The question whether it also solves the above variant of the problem of easy knowledge is trickier. The conclusion of the argument in that case is not that an epistemic rule (going by the gas gauge) is reliable, but rather that it is super-reliable. So, NRC might be said not to apply, and hence to be too weak.

However, a seemingly minor alteration is enough to make it apply. Indeed, Weisberg (2009, p. 5) slightly misquotes NRC: as he states the principle, the first sentence reads, "A belief about the reliability of rule $R$ cannot be justified by the application of $R$," instead of "A belief that an epistemic rule $R$ is reliable cannot be justified by the application of $R$," which is what Vogel has. In Weisberg's version of it, NRC does apply to the super-reliable gauge case, and it does give the correct verdict about it. After all, the rule of going by the gas gauge serves to establish a conclusion about the reliability of that rule (to wit, that it is super-reliable). So let us suppose for now that Vogel agrees with the suggested version of NRC (as will become apparent shortly, he also has reason to disagree with it). Even so, argues Weisberg, the principle must be rejected, for it is still too weak; and, what is worse, it is not just too weak, but also too strong.

Weisberg's claim that NRC is too weak is based on an example in which a person

knows The New York Times to be reliable every day of the week, though less reliable on Sundays than other days. Not knowing the day of the week, he reads the paper front to back and comes to believe of each sentence that the Times says it and it is true. He concludes that today is not Sunday, since the Times is normally not accurate on Sundays. Weisberg (2009, p. 6f)

The person's reasoning appears to be fallacious. ${ }^{5}$ Yet the conclusion he draws is not that some epistemic rule is reliable - in fact, it is not about reliability at all —and so NRC does not apply. Hence, to the extent that it is reasonable to think there must be a unitary explanation of what is wrong with the problem of easy knowledge and with the above New York Times case, NRC is too weak indeed.

To argue that NRC is also too strong, Weisberg presents the case of a person who knows The New York Times to be reliable and reads an article in The New York Times

\footnotetext{
5 We note in passing that Weisberg must be supposing that the Times does not issue warnings to the effect that a given edition is more likely to contain errors than normal. Suppose, for instance, that the lower than normal reliability of the Sunday Times is due to the fact that more technical problems in the production process occur on Saturday nights than on other nights of the week-perhaps because some of the technicians like to go out drinking on Saturday evenings before coming to work. The Sunday edition of the newspaper might then frequently contain a warning of the said kind. If the person in the case Weisberg considers has regularly encountered such warnings in the Times on Sundays, and knows about the policy of issuing this kind of warning, then there might be nothing wrong with his reasoning. But we grant the (implicit) supposition that no such policy exists. Note also that Weisberg must be assuming (at a minimum) that the sentences the person reads and comes to believe are jointly consistent (and hence also self-consistent). Otherwise it would be immaterial whether NRC can handle this case, as no appeal to that principle would be necessary to explain what is wrong with the person's reasoning.
} 
reporting three independent studies which found this newspaper to be significantly more reliable than most of its readers think. On this basis, she comes to believe that the newspaper is even more reliable than she thought it was. There seems to be nothing wrong with this person's reasoning, and her conclusion seems to be justified. Yet, Weisberg thinks, according to NRC it is not justified: "Her rule is to trust the Times, and she applies that rule in the reasoning that leads her to believe the Times is more reliable than she thought" (p. 6).

Here, the exact version of NRC is crucial again. In Vogel's original version, NRC does not apply to this case: that the Times is more reliable than the person thought it was - the conclusion she draws - is certainly a conclusion about the reliability of the Times, but it is not the conclusion that the Times is reliable. The case would thus fail to establish that NRC, as intended by Vogel, is too strong. As we saw above, however, there is also pressure on Vogel to adopt Weisberg's version of NRC, lest the principle be too weak. So, whichever version of the principle Vogel agrees to settle on, NRC is found wanting either way.

Weisberg presents one further case in his paper which is meant to show that the problem of easy knowledge has a counterpart even for probabilistic inferences. In this case, again, a person knows that the Times is reliable. After reading the first sentence, $P$, of today's edition of the paper, she comes to believe that the Times says $P$ and $P$ is true. She thereupon becomes slightly more confident that the next sentence, $Q$, will be true as well. Nevertheless, Weisberg contends, even this slight gain in confidence is intuitively impermissible on the basis of her reasoning.

Weisberg then comes to his own proposal by noting a common pattern in the reasoning that is going on in the problem cases. All these cases involve what he terms "lemmistic reasoning" (p. 10). The persons in these cases all first draw preliminary conclusions- "lemmas"-from premises (data, observations) and then use these preliminary conclusions to infer their final conclusion from the premises. Moreover, in each of the arguments the premises alone fail to probabilistically support the conclusion, yet when combined with the preliminary conclusions that are drawn from them they do probabilistically support the conclusion.

This leads Weisberg to postulate the following defeater for inductive reasoning (p.10):

No Feedback (NF) If (i) $C$ is inferred from $L_{1}-L_{n}$ (and possibly other premises)

by an argument whose justificatory power depends on making $C$ at least $x$ probable, (ii) $L_{1}-L_{n}$ are inferred from $P_{1}-P_{n}$, and (iii) $P_{1}-P_{n}$ do not make $C$ at least $x$ probable without the help of $L_{1}-L_{n}$, then the argument for $C$ is defeated.

It is not hard to see that NF gives the right verdict in the problem cases. For instance, both in Vogel's original case and in the case of the super-reliable gauge, the gauge readings on their own do not do anything to make it more probable that the gauge is reliable, even if in combination with the preliminary conclusions that are being drawn from them-that the tank contains the amount of gas that the gauge indicates- they do. NF also gets right the case where a person infers that the Times is even more reliable than she thought it was from reading an article in the Times; it countenances that conclusion inasmuch as the results reported in the article on their own, without the help of any "lemmas," suffice to make the conclusion highly probable. 
Still, not all is well for NF. In particular, we believe that the principle is too strong. First, notice that we cannot do away with the parenthetical "and possibly other premises" in clause (i) of NF. For instance, in the original problem of easy knowledge and in the case of the super-reliable gauge, the $L_{i}$ are the preliminary conclusions that there is such-and-such an amount of fuel in the gas tank, which are drawn from the gauge readings (the $P_{i}$ ). In these cases, the ultimate conclusion that the gauge is reliable (or super-reliable) is certainly not based on the $L_{i}$ alone. In terms of these schematic letters, in the said arguments each $L_{i}$ is conjoined with the corresponding $P_{i}$ from which it was inferred, and these conjunctions then together serve as premises for an inductive argument. Or, clearer still, ${ }^{6}$ consider the Sunday Times case, where the conclusion - that today is not Sunday-is also inferred from the premise (supposed to be part of the person's background knowledge) that the Times is less reliable on Sundays than on other days of the week. Thus, without reference to possible other premises next to the $L_{i}$ being used in the inference of the ultimate conclusion, NF would apply to none of these cases.

On the other hand, with the reference to those other premises NF in effect invalidates what would seem to be quite uncontestable forms of scientific reasoning. Just consider any case where we derive a number of laws from some given data sets and then base a prediction on those laws plus initial conditions, and where the union of the data sets alone does not make the prediction probable, but the laws together with the initial conditions do.

For example, let $C$ be the prediction that there will be a total eclipse of the sun in Argentina on July 11, 2020. The prediction may well be (and, let us suppose, is) inferred from Kepler's first two laws of planetary motion-which we are free to identify with the $L_{i}$ in Weisberg's schema-and other premises, like premises concerning the locations of the sun, the moon, and the earth at earlier points in time. It can plausibly be assumed that, based on this inference, the scientists are quite certain that the prediction is correct. Now, Kepler famously inferred his first two laws from numerous (for that time extremely accurate) observations of Mars that had been made by Tycho Brahe; say that the relevant observations for law $L_{i}$ were $P_{i}$, for $i \in\{1,2\}{ }^{7}$ Still, these observations alone should not be expected to make the solar eclipse even highly probable. Indeed, without the designated initial conditions, it will be impossible to infer the eclipse from Kepler's laws. So, according to NF, the prediction is based on defective reasoning. But, for all we know, the reasoning by which the scientists arrived at their prediction is perfectly sound.

An even more striking example, in which the premises and conclusion belong to ostensibly unrelated areas of physics, may be the following. In 1925, Pauli inferred his well-known Exclusion Principle, according to which no two electrons in an atom can have the same four quantum numbers, from measurements of frequencies of spectral

\footnotetext{
${ }^{6}$ Clearer, because in the gas gauge cases one could, strictly speaking, identify the $L_{i}$ with the conjunctions "The gauge says the tank is $x$ percent full and the tank is $x$ percent full" (though Weisberg $(2009$, p. 11) himself identifies the $L_{i}$ with the second conjuncts of those conjunctions).

7 In this case the $P_{i}$ can be plausibly thought of as being conjunctions of statements reporting locations of Mars at different points in time. It may be worth noting that nothing in NF prevents that $P_{i}=P_{j}$ for some, or even all, $i$ and $j$.
} 
lines. Three years later, Heisenberg used this principle, together with other premises (such as the time-independent Schrödinger equation), to explain the phenomenon of ferromagnetism (the phenomenon that some materials can be magnetized by placing them in a magnetic field). Identifying $L$ with Pauli's principle and $P$ with the measurement results concerning spectral lines, we have a situation in which a conclusion $C$ concerning ferromagnetism is inferred from $L$ together with other premises, where $L$ is inferred from $P$, but where $P$ does not make $C$ highly probable, and may even be probabilistically irrelevant to $C$. Again, NF would seem to rule against what scientists generally regard as a perfectly sound $\operatorname{argument}^{8}$

Rather than querying whether NF can be modified in such a way that it does permit the aforementioned type of scientific reasoning, we would like to draw attention to the somewhat surprising fact that a solution to the problem of easy knowledge (as well as to Weisberg's later problem cases) could already be found in the literature at the time the problem was first presented by Vogel. Like Weisberg's proposal, the solution we have in mind locates the fault in the type of reasoning employed in the problem cases, but it rules out these cases much more selectively than NF does, and countenances for instance the inference of place and date of the solar eclipse in the way described above. We now turn to this solution.

\section{Bootstrapping done properly}

The question we are concerned with-the question of what is precisely wrong with the type of reasoning involved in the problem cases considered above-really belongs to confirmation theory, or, more generally, the theory of non-deductive reasoning. For, patently, these cases all involve the performing of experiments-checking gas gauges, reading sentences in a newspaper-and the drawing of conclusions that do not follow deductively from the registered outcomes of the experiments. ${ }^{9}$ The type of "lemmistic" reasoning that Weisberg correctly identifies as being involved in the problem cases of easy knowledge is not at all unknown to confirmation theorists, although they have used the term "auxiliary" (or "auxiliary hypothesis") for what Weisberg calls "lemma." In this final section, we look at Glymour's (1980a) confirmation theory, which provides a more extensive and illuminating treatment of the import and role of the use of auxiliaries than any other confirmation theory to date. In particular, we would like to highlight what we take to be a fundamental confirmation-theoretic truth captured by

\footnotetext{
8 We owe this example to Fred Muller.

${ }^{9}$ It would be a mistake to think that confirmation-theoretic considerations are only relevant to scientific reasoning (though an understandable mistake, perhaps, as confirmation theory is nowadays commonly regarded as being a branch of the philosophy of science) and not to the reasoning involved in answering such mundane questions as how much fuel there is in the tank or whether today is Sunday. As Hempel (1945, p. 7) in his pioneering work on confirmation theory already remarked, "an analysis of confirmation is of fundamental importance also for the study of the central problem of what is customarily called epistemology; this problem may be characterized as the elaboration of 'standards of rational belief' or of criteria of warranted assertibility." Equally, Bayesians famously define the confirmation relation in terms of rational graded belief that they intend to apply quite generally, not only to scientists, and not only insofar as scientific hypotheses are concerned.
} 
this theory and argue that it is the key to solving the problem of easy knowledge, both in its original form and in Weisberg's variants of it.

It is still sometimes loosely said that confirmation theories try to specify for every evidence sentence $E$ and every hypothesis $H$ the conditions under which $E$ is evidence for $H$. However, it is regarded as one of the major lessons of twentieth-century philosophy of science that, contrary to what the foregoing formulation suggests, the confirmation relation is really three-place rather than two-place, in that evidence generally accrues to a hypothesis only relative to one or more auxiliary hypotheses. One very straightforward reason for this is that in taking a set of data to constitute evidence for or against a given hypothesis, one must rely on the auxiliary hypothesis that the devices used for obtaining the data were working properly. More interesting reasons can be found in virtually any introductory text on confirmation theory.

The involvement of auxiliaries in hypothesis testing can easily make it seem as if there is only relative confirmation (confirmation relative to one or more auxiliaries). Some even took it to motivate a rampant epistemic relativism. ${ }^{10}$ But in his Glymour (1980a), Glymour forcefully argues that the indispensability of auxiliaries does not block the possibility of absolute (non-relative) confirmation. The central idea is that, while the testing of single hypotheses will typically require the help of auxiliaries, theories, thought of as complexes of hypotheses, may still be confirmed in an absolute sense. Piecemeal relative confirmation of the separate hypotheses comprised by a theory (the theory's axioms, or laws, if one likes) adds up, subject to the satisfaction of a number of requirements, to absolute confirmation of that theory as a whole. ${ }^{11}$

To unpack this idea, Glymour (1980a, Chap. 5) proposes what he calls a bootstrapping account of confirmation. Consider a theory $T=\left\{H_{1}, \ldots, H_{n}\right\}$, where the $H_{i}$ are the axioms (or laws) of $T$. Then a first, obvious, requirement on Glymour's account for an evidence sentence $E$ to bootstrap confirm $T$ is that $E$ be consistent with $T$. More substantially, for each $H_{i}, E$ must confirm $H_{i}$ relative to a (possibly empty) set $T^{*}$ of auxiliaries, where it must hold that $T^{*} \subseteq T$. Finally, there must be an evidence sentence $E^{\prime}$ such that (i) the test that yields (or yielded) $E$ could yield (or could have yielded) $E^{\prime}$, and (ii) $E^{\prime}$ disconfirms $H_{i}$ relative to $T^{*}$, that is, relative to exactly those auxiliaries relative to which $E$ confirms $H_{i}$.

The definition of bootstrap confirmation is intended to be neutral as regards the interpretation of the three-place relations of confirmation and disconfirmation referred to in the last two conditions. Although in his own presentation of bootstrap confirmation Glymour understands these relations in terms of Hempel's (1943) positive instance account, he stresses that bootstrap confirmation is not wedded to this confirmation theory, and that the said relations can, for instance, also be interpreted along hypothetico-deductive or probabilistic lines. ${ }^{12}$

\footnotetext{
10 As expressed, for instance, in Quine (1951, p. 43) famous dictum that "[a]ny statement can be held true come what may, if we make drastic enough adjustments elsewhere in the system." See Douven (2011) for more on this.

11 It is worth mentioning that Glymour's (1980a) presentation of the bootstrapping account leaves some room for interpretation. Our interpretation of it accords with that of Earman and Salmon (1992, p. $52 \mathrm{ff}$ ) and Christensen (1997, p. 372).
}

12 See Glymour (1980a, p. 127). Glymour (1980b) and Douven and Meijs (2006) present probabilistic versions of bootstrap confirmation theory. 
Bootstrapping, as defined by Glymour's account, is lemmistic reasoning with a vengeance. "Lemmas" are confirmed by the data possibly with the help of further (not necessarily different) lemmas, which in their turn may be confirmed by the data with the help of possibly some lemmas they helped to confirm, resulting, if all goes well, in a confirmation of the "conclusion"- - a theory-by the data. What is more, the bootstrapping account clearly sanctions what might strike one as a form of epistemically circular reasoning: it is perfectly fine, as far as the above definition goes, that in testing a theory one makes use of auxiliaries that are part of that very theory; at least this is perfectly fine as long as certain requirements are met. As several authors, most notably Vogel, have thought the problem of easy knowledge to be, at root, a problem stemming from epistemic circularity, one might wonder how an appeal to Glymour's theory of bootstrap confirmation could offer any help with tackling that problem.

The crux is that, on Glymour's account, bootstrapping does not license an unrestricted use of auxiliaries in testing theories. The most important restriction, for our purposes in any event, is the one given by the third condition above, which is also known as the "nontriviality clause." It is motivated by this principle:

No Risk, No Gain (NRNG) "To test a hypothesis we must do something that could result in presumptive evidence against the hypothesis" (Glymour (1980a, p. 115)).

A bit less succinctly, a hypothesis cannot gain support in a test unless that test puts the hypothesis at risk of being disconfirmed; if, by its very design — which includes the choice of auxiliaries to be relied on-a test ensures confirmation, whatever the data may be, then that test is trivial (not a real test at all) and the confirmation it ensures spurious. Glymour's account rules out such cases of spurious confirmation by requiring that the tests for the separate hypotheses constituting a theory do not shield these hypotheses from disconfirmation.

With the aid of many examples from the practice of science, Glymour argues that bootstrap confirmation is an utterly common form of scientific reasoning. While it would be an exaggeration to say that Glymour's theory has been received to universal acclaim (see below), the nontriviality clause, or rather the insight that lies at its root-as embodied in the principle NRNG - has not been challenged. This should be unsurprising, as it appears to be hardly more than a truism. ${ }^{13,14}$ Even so, this insight will help us see where the various arguments occurring in the problem cases of easy knowledge go awry. On our diagnosis, the flaw present in all these arguments is precisely that they fail to meet the requirement NRNG imposes on testing procedures.

\footnotetext{
${ }^{13}$ For instance, Giere, who otherwise does not seem to share Glymour's views on confirmation, appeals to the principle in defending the view that a hypothesis explicitly constructed to account for certain data does not receive any support from those data: "If the known facts were used in constructing the model and were thus built into the resulting hypotheses ... then the fit between these facts and the hypothesis provides no evidence that the hypothesis is true [as] these facts had no chance of refuting the hypothesis" (Giere 1984, p. 161).

14 Considering that shielding a hypothesis from disconfirmation will often be a gradual rather than a categorical matter, even a generalization of NRNG according to which a test is more telling the less it shields the hypothesis from disconfirmation would seem plausible; see Douven and Meijs (2006, p. 108) on degrees of trivialization. This principle is hard or even impossible to incorporate in Glymour's qualitative theory of bootstrap confirmation, but it is formally captured by Douven and Meijs's (2006, p. 109) measure of bootstrap confirmation.
} 
This is immediately obvious in the problem of easy knowledge as presented in Vogel (2000) and in Weisberg's super-reliable gas gauge case: the persons in these cases accept any gauge reading as being correct (that is, as registering the actual level of gas in the tank). It is plausible to think of the persons as relying, if only tacitly, on the auxiliary that the gas gauge is reliable. But the important point is that, in both cases, disconfirmation of the hypothesis at issue - that the gas gauge is reliable, respectively, super-reliable - is precluded. So long as the testing procedure that delivers the evidence sentences in these cases is held fixed, it cannot deliver a sentence that disconfirms the reliability (or super-reliability) of the gas gauge. As the target hypotheses are never at risk of being disconfirmed in the tests performed, no confirmation is obtained in either of these cases. That follows from NRNG. ${ }^{15}$

The same holds for the case which Weisberg uses to argue that Vogel's principle $\mathrm{NRC}$ is too strong. In this case, recall, a person infers that today is not Sunday from the intermediate conclusion that today's issue of the Times is accurate and his antecedent knowledge that the Times is reliable, though less reliable on Sundays than other days. Here, too, the test this person uses to arrive at the said intermediate conclusion is designed in such a way that disconfirmation of that hypothesis - that today's newspaper is accurate - is ruled out from the start. Of any sentence he reads he concludes, relying on the auxiliary hypothesis that the Times is reliable, that it is true-whatever the sentence says. Consequently, no evidence he could obtain by this testing procedure could disconfirm that today's Times is accurate.

Weisberg's final problem case, the case in which a person becomes slightly more confident that the second sentence, $Q$, of today's newspaper is true after concluding that the first sentence, $P$, is true, needs a bit more careful consideration. Literally, the case reads as follows:

Starla knows that the Times is reliable. She opens today's paper, reads the first sentence, $P$, and comes to believe that the Times says $P$ and $P$ is true. She then ever so slightly increases her estimate of her epistemic probability that the next sentence, $Q$, is true. (Weisberg $(2009$, p. 8f))

Notice that, as far as this specification of the case goes, the person may for intuitively perfectly good reasons have raised her probability for $Q$. Suppose, for instance, $P$ is

\footnotetext{
15 But what if we slightly altered the gauge cases by presuming that the gauges may start to flicker when they malfunction? The persons' reasoning would not be any better in those cases, yet now-one might claim-disconfirmation of the hypothesis that the gauge is reliable, respectively super-reliable, is possible (the gauge could start to flicker). One thing one could say here is that if a gauge has the said feature, then the hypothesis that it is reliable amounts to the two-part claim that (i) it correctly indicates the level of fuel in the tank whenever it is not flickering and (ii) it malfunctions whenever it is flickering. Thus understood, proceeding in the way the persons in the gauge cases do could still not yield disconfirmation of the said hypothesis. In addition to this, we point to the fact that, as said in note 14, a generalization of NRNG is defensible. In light of this, one would expect that cases can be constructed that involve an intuitively inadmissible form of bootstrap reasoning even though no violation of NRNG occurs in them, namely, cases in which a hypothesis is largely, even if not completely, shielded from disconfirmation, and so the test performed is not very telling, while the person at issue in the case is still said to acquire, on the basis of that test, warrant for, or even knowledge of, the hypothesis. A suitable generalization of NRNG, for instance along the lines of Douven and Meijs (2006, p. 108) thesis that "the higher the 'degree of triviality' of a test of a particular hypothesis is, the less that test can contribute to the degree of bootstrap support the theory as a whole obtains," would help to prevent the ascription of warrant and, a fortiori, knowledge, in such cases.
} 
a sentence saying that several studies have shown the Times to be more reliable than most of its readers think. Then surely no one would object against Starla's slightly increasing her confidence that the next sentence will be true. Conversely, suppose that $Q=\neg P$, or that $P$ is the first sentence of an editorial saying that second sentences of editorials have been found to be relatively often false. In either case, no one will have difficulty accounting for what is wrong with Starla's reasoning. It is clear, though, that this is not how Weisberg wants us to understand the case. The idea, no doubt, is that the person increases her confidence in $Q$ purely on the basis of her conclusion that the Times says $P$ and $P$ is true, coupled with the fact that the sentence $Q$ is printed in the Times; neither the content of $P$ nor that of $Q$ matters in any of this. But thus understood, this case, too, involves a violation of NRNG. After all, the person then seeks probabilistic confirmation for the hypothesis that a given sentence is true by reading another sentence, where she takes her background knowledge that the Times is reliable to license the conclusion that this other sentence is true, regardless of what it says. Again, no disconfirmation can occur in this test, so the test is trivial and cannot yield confirmation either.

Finally, NRNG is manifestly not violated in the case which Weisberg took to show that NRC is too strong, and which was explicitly meant to exhibit a form of lemmistic reasoning that is unproblematic. Recall that in this case a person knowing that the Times is reliable concludes that it is even more reliable than she thought from reading a report in the paper saying that the Times is more reliable than most readers think. This was said to be unproblematic indeed, and Glymour's confirmation theory, or NRNG, does not suggest otherwise. The fact that the person uses her background knowledge about the reliability of the Times as an auxiliary in obtaining confirmation for the hypothesis that the paper is even more reliable than she thought does not preclude that she receives evidence against that hypothesis, given that the article might have said that studies show that the Times is less reliable than most readers think, or even that it is not reliable at all.

To sum up our case against Weisberg's response to the problem of easy knowledge: while we do not dispute the effectiveness of his response, in particular of his proposal NF, we think it is needlessly restrictive. That it rules out forms of reasoning that seem perfectly legitimate and useful might perhaps be acceptable as a last resort, if we had no other option of dealing with the problem of easy knowledge. But, as we hope to have shown above, appeal to a principle that is already to be found in the confirmationtheoretic literature, and that appears plausible if any principle does, solves the problem of easy knowledge as well, but in a much more discriminating way. In particular, it does not rule out the kind of utterly normal scientific reasoning considered at the end of the previous section, nor, to the best of our knowledge, any other form of reasoning deemed pre-theoretically valid.

We end with three comments. First, Vogel (2000, p. 615) briefly considers the possibility that what might go wrong in his gas gauge case is that the person uses a procedure that "could not have possibly yielded any other result other than the one it did, namely, that the gauge is reliable." But he rejects this possibility, for " $[\mathrm{t}]$ he process by which I know I am conscious when I am is surely a reliable one, yet that process could not return a verdict other than that I am conscious" (ibid.). Vogel leaves it at that, but the point, put in our terms, is clearly that NRNG has counterexamples, so that an appeal 
to it is unavailing. Note, however, that the hypothesis "I am conscious" seems to be of a very different kind than, for instance, the hypothesis that the gas gauge of one's car is reliable. The former seems to be no more open to empirical testing (nor more open to doubt) than "I exist" is. Neither is necessarily true, yet they cannot consistently be denied; they are what some have termed pragmatic tautologies. So, one thing we could say is that "I am conscious" is not even within the scope of, and hence cannot refute or cast doubt on, Glymour's account of bootstrap confirmation or NRNG, which manifestly pertain to empirical claims only, not to tautologies, pragmatic or otherwise. Alternatively, if one insists on classifying the hypothesis as being empirical (given that it is contingent), we could say that if, according to a given confirmation theory, no evidence can confirm a hypothesis that is undeniable anyway, that is hardly a blemish for that confirmation theory, and certainly no reason to abandon it.

Second, it may have been noted that Glymour's bootstrapping account of confirmation sanctions the use of a hypothesis as an auxiliary in its own test or tests. As Glymour and various other authors have emphasized, this does not by itself trivialize such tests. Indeed, as we just saw, one may assume that the Times is reliable in testing that very assumption, only to read an article in the Times saying that it is not reliable. Still, Christensen (1983), Edidin (1983), and van Fraassen (1983) derived some untoward consequences from Glymour's theory that all essentially depend on using a hypothesis as an auxiliary in its own test. In response to these criticisms, Glymour then restricted the auxiliaries admissible in a test to hypotheses other than the one being tested. ${ }^{16}$ This amounts to imposing something like Vogel's original version of NRC on the above definition of bootstrap confirmation. As we noted, however, there is nothing wrong with NRC in itself. It appeared too weak to deal successfully with all problem cases presented by Weisberg, but that is fine, given that it is not needed for blocking any of them; appeal to NRNG suffices perfectly for that.

Third, the main message of our paper is that bootstrapping, or lemmistic reasoning, or drawing on auxiliaries in testing a theory even if the auxiliaries are part of that theory, does not have any intrinsic defects. It is just that certain forms of bootstrapping must be banned, namely those that would lead to cases of spurious confirmation, those, that is, that violate NRNG. What, in the nature of reliabilism, could keep the reliabilist from subscribing to this? As far as we can presently see, disagreement about RT should have no implications for one's views about the canons of non-deductive reasoning, about which inferences can be validly made on the basis of a given set of data, or about which uses of auxiliaries are permissible and which are not. Be that as it may, the principle NRNG should, as intimated, appear uncontentious from any perspective. As a result, there is nothing distinctively internalist to our solution of the problem of easy knowledge.

Acknowledgments Thanks are due to Jonathan Adler, Christopher von Bülow, and the participants at a meeting of the European Epistemology Network in Brussels for very helpful comments on ancestors of this paper.

$\overline{16}$ See Earman and Glymour (1988). 
Open Access This article is distributed under the terms of the Creative Commons Attribution License which permits any use, distribution, and reproduction in any medium, provided the original author(s) and the source are credited.

\section{References}

Bergmann, M. (2004). Epistemic circularity: Malignant and benign. Philosophy and Phenomenological Research, 69, 709-727.

Christensen, D. (1983). Glymour on evidential relevance. Philosophy of Science, 50, 471-481.

Christensen, D. (1997). What is relative confirmation? Nô̂s, 31, 370-384.

Cohen, S. (2002). Basic knowledge and the problem of easy knowledge. Philosophy and Phenomenological Research, 65, 309-329.

Douven, I. (2002). A new solution to the paradoxes of rational acceptability. British Journal for the Philosophy of Science, 53, 391-410.

Douven, I. (2007). A pragmatic dissolution of Harman's paradox. Philosophy and Phenomenological Research, 74, 326-345.

Douven, I. (2011). Relativism and confirmation theory. In S. Hales (Ed.), The Blackwell companion to relativism (pp. 242-265). Oxford: Blackwell.

Douven, I., \& Meijs, W. (2006). Bootstrap confirmation made quantitative. Synthese, 149, 97-132.

Douven, I., \& Williamson, T. (2006). Generalizing the lottery paradox. British Journal for the Philosophy of Science, 57, 755-779.

Dretske, F. (1970). Epistemic operators. Journal of Philosophy, 67, 1007-1023.

Earman, J., \& Glymour, C. (1988). What revisions does bootstrap testing need? Philosophy of Science, 55, 260-264.

Earman, J., \& Salmon, W. (1992). The confirmation of scientific hypotheses. In M. Salmon (Ed.), Introduction to the philosophy of science (pp. 42-103). Upper Saddle River, NJ: Prentice Hall.

Edidin, A. (1983). Bootstrapping without bootstraps. In J. Earman (Ed.), Testing scientific theories (pp. 43-54). Minneapolis, MN: University of Minnesota Press.

Giere, R. (1984). Understanding scientific reasoning (2nd ed.). New York: Holt, Rinehart, and Winston.

Glymour, C. (1980a). Theory and evidence. Princeton, NJ: Princeton University Press.

Glymour, C. (1980b). Bootstraps and probabilities. Journal of Philosophy, 67, 691-699.

Hempel, C. G. (1943). A purely syntactical definition of confirmation. Journal of Symbolic Logic, 8, $122-143$.

Hempel, C. G. (1945). Studies in the logic of confirmation (I). Mind, 54, 1-26.

Quine, W. V. O. (1951). Two dogmas of empiricism. Philosophical Review, 60, 20-43. (Reprinted in his From a logical point of view (pp. 20-46). Cambridge, MA: Harvard University Press; the page reference is to the reprint).

Van Cleve, J. (2003). Is knowledge easy or impossible? Externalism as the only answer to the skeptic. In S. Luper (Ed.), The skeptics (pp. 45-59). Aldershot: Ashgate.

van Fraassen, B. C. (1983). Theory comparison and relevant evidence. In J. Earman (Ed.), Testing scientific theories (pp. 27-42). Minneapolis, MN: University of Minnesota Press.

Vogel, J. (2000). Reliabilism leveled. Journal of Philosophy, 97, 602-623.

Vogel, J. (2008). Epistemic bootstrapping. Journal of Philosophy, 105, 518-539.

Weisberg, J. (2009). Bootstrapping in general. prizewinning essay Young Epistemologist's Prize 2009. (Available at: http://www.utm.utoronto.ca/ weisber3/docs/Intransitivityv2.pdf).

Zalabardo, J. (2005). Externalism, skepticism, and the problem of easy knowledge. Philosophical Review, 114, 33-61. 